\title{
Can Waterpipe Smoking cause Oral Cancer?
}

\author{
${ }^{1}$ A Thirumal Raj, ${ }^{2}$ Shankargouda Patil, ${ }^{3}$ Kamran H Awan, ${ }^{4}$ Chandini Rajkumar
}

How to cite this article: Raj AT, Patil S, Awan KH, Rajkumar C. Can Waterpipe Smoking cause Oral Cancer? World J Dent 2018;9(1):1.

\section{Source of support: Nil \\ Conflict of interest: None}

The World Health Organization (WHO) study group on Tobacco Product Regulation had suggested a striking similarity between waterpipe smoking (WPS) and cigarette smoking. Several well-established carcinogens present in cigarettes like polycyclic aromatic hydrocarbons, volatile aldehydes, phenols, and heavy metals were also noted in WPS. ${ }^{1-3}$ Further, the period of one WPS session is significantly higher than smoking one cigarette. This increased exposure time causes a potentially increased intake of smoke and other chemical components of WPS. ${ }^{2}$ The difference in the dosage of WPS and cigarette smoking is so significant that the WHO has equated 1 WPS session to smoking more than 100 cigarettes. $^{3}$

Sajid et al ${ }^{4}$ estimated the carcinoembryogenic antigen (CEA) levels among waterpipe smokers and nonsmokers. The WPS users had a mean CEA level of $3.58 \pm 2.61 \mathrm{ng} / \mathrm{mL}$ in comparison to $2.35 \pm 1.15 \mathrm{ng} / \mathrm{mL}$ in nonsmokers. Mamtani et $\mathrm{al}^{5}$ published a meta-analysis in 2016 analyzing the relationship between cancer and WPS. The meta-analysis showed a strong correlation between WPS and cancer of the lung, head and neck, and esophagus. A systematic review conducted by Awan et al ${ }^{6}$ observed WPS to have a strong correlation with lung cancers, but other forms of cancers were found to show a relatively weak to no association. At present, the association between WPS and

\footnotetext{
${ }^{1}$ Department of Oral Pathology and Microbiology, Sri Venkateswara Dental College and Hospital, Chennai, Tamil Nadu, India

${ }^{2}$ Division of Oral Pathology, Department of Maxillofacial Surgery and Diagnostic Sciences, College of Dentistry, Jazan University Jazan, Kingdom of Saudi Arabia

${ }^{3}$ Department of Oral Medicine, College of Dental Medicine Roseman University of Health Sciences, South Jordan, Utah, USA

${ }^{4}$ Department of Oral Pathology and Microbiology, Sathyabama University Dental College and Hospital, Chennai, Tamil Nadu, India

Corresponding Author: A Thirumal Raj, Department of Oral Pathology and Microbiology, Sri Venkateswara Dental College and Hospital, Chennai, Tamil Nadu, India, Phone: +918122627810, e-mail: thirumalraj666@gmail.com
}

oral cancer is poorly documented. Most studies exploring the effects of WPS and oral cancer have failed to account for the concomitant use of cigarette, tobacco, and alcohol. ${ }^{7}$ Thus, as these studies were biased, it was not possible to access WPS as an independent risk factor for oral cancer. ${ }^{8}$

It is well established that cigarette smoking can cause malignant changes in epithelial cells and several studies have shown cigarette and WPS to share several carcinogens. ${ }^{1}$ In addition to this, as stated earlier, WHO has equated 1 WPS session to smoking 100 cigarettes or more. ${ }^{3}$ Based on the above data, it can be hypothesized that WPS is potentially carcinogenic to the oral mucosa. The future research goals in WPS include conduction of multicenter case-control and prospective studies with adequate sample size to test this hypothesis. To record exposure times to allow estimation of dose-response. Further, it is vital to conduct in vivo and in vitro studies to elicit the molecular changes in the oral mucosa from chronic exposure to WPS. Decoding the molecular biology of WPS induced carcinogenesis will provide early diagnostic markers and vital therapeutic targets.

\section{REFERENCES}

1. AklEA, GunukulaSK, AleemS, Obeid R, JaoudePA,Honeine R, Irani J. The prevalence of waterpipe tobacco smoking among the general and specific populations: a systematic review. BMC Public Health 2011 Apr;11:244.

2. Primack BA, Carroll MV, Weiss PM, Shihadeh AL, Shensa A, Farley ST, Fine MJ, Eissenberg T, Nayak S. Systematic review and meta-analysis of inhaled toxicants from waterpipe and cigarette smoking. Public Health Rep 2016 Jan-Feb;131(1):76-85.

3. WHO study group on Tobacco Product Regulation (TobReg). Advisory Note. Waterpipe tobacco smoking: health effects research needs and recommended actions by regulators. Geneva: WHO; 2005.

4. Sajid KM, Chaouachi K, Mahmood R. Hookah smoking, and cancer: carcinoembryonic antigen (CEA) levels in exclusive/ ever hookah smokers. Harm Reduct J 2008 May;5:19.

5. Mamtani R, Cheema S, Sheikh J, Mulla A, Lowenfels A, Maisonneuve P. Cancer risk in waterpipe smokers: a metaanalysis. Int J Public Health 2016 Jan;62(1):73-83.

6. Awan KH, Siddiqi K, Patil S, Hussain QA. Assessing the effect of waterpipe smoking on cancer outcome-a systematic review of current evidence. Asian Pac J Cancer Prev 2017 Feb;18(2):495-502.

7. Al-Amad SH, Awad MA, Nimri O. Oral cancer in young Jordanians: potential association with frequency of narghile smoking. Oral Surg Oral Med Oral Pathol Oral Radiol 2014 Nov;118(5):560-565.

8. Raj AT, Patil S, Sarode S, Sarode G. Oral health hazards of water pipe smoking: current evidence. J Contemp Dent Pract 2017 Jul;18(7):539-540. 